\title{
On the Poincaré Algebra in a Complex Space-Time Manifold
}

\author{
Nathalie Debergh ${ }^{*}$, Gilles D'Agostini², Jean-Pierre Petit ${ }^{2}$ \\ ${ }^{1}$ Department of Agronomy, Haute Ecole Charlemagne, Huy, Belgium \\ ${ }^{2}$ Manaty Research Group, Glanon, Dijon, France \\ Email: *nathalie.debergh@hech.be
}

How to cite this paper: Debergh, N. D’Agostini, G. and Petit, J.-P. (2021) On the Poincaré Algebra in a Complex Space-Time Manifold. Journal of Modern Physics, 12, 218-228.

https://doi.org/10.4236/jmp.2021.123017

Received: December 27, 2020

Accepted: February 7, 2021

Published: February 10, 2021

Copyright () 2021 by author(s) and Scientific Research Publishing Inc. This work is licensed under the Creative Commons Attribution International License (CC BY 4.0).

http://creativecommons.org/licenses/by/4.0/

(c) (i) Open Access

\begin{abstract}
We extend the Poincaré group to the complex Minkowski space-time. Special attention is paid to the corresponding algebra that we achieve through matrices as well as differential operators. We also point out the generalizations of the two Casimir operators.
\end{abstract}

\section{Keywords}

Complex Minkowski Manifold, Poincaré Group, Lie Algebraic Methods Applied to Physics

\section{Introduction}

Complex numbers are known to be powerful mathematical tools to describe physical phenomena. Usually, their role is limited to intermediate calculations and they are not welcome in final results. However, sometimes these results do depend on these numbers in the sense that they explicitly appear in the final equations.

To our knowledge, complex energies first appeared as intrinsic components of the physical paradigm in the relativistic Kemmer-Duffin-Petiau equation [1], dealing with vector mesons. When they are subject to a sufficiently strong magnetic field $\left(B>\frac{m^{2}}{e}\right)$, their energies, whose squares are given by ${ }^{1}[2]$

$$
E^{2}=m^{2}+2 e B\left(n+\frac{1}{2}+s\right) ; n=0,1,2, \cdots ; s=0, \pm 1
$$

become purely imaginary ones.

More recently, some cosmological models [3] used an imaginary time. They

${ }^{1}$ Here and all along this paper, we take the velocity of light equal to 1 . 
all conjecture a join between areas of imaginary time and areas of real time. Somehow, real time emerges from imaginary time, the latest being helpful to remove gravitational singularities.

This idea is clearly not unanimous because an imaginary time is most of the time seen as non-physical, even heretical. Also voices [4] rose to emphasize the inconsistency of such a transition between imaginary and real times which still remains vague. Some attempts have been performed to specify it. For instance, in [5], imaginary time stands before the Planck time and is related to imaginary energies seen as pure information. Thus, an imaginary time is compatible with the fact that time does not go by and the information, which in a sense, replaces matter, is retained until time rotates and becomes real. To cite the authors of [5], this imaginary time is analogous to the moment when the music is burned on a CD but not yet listened to. It can wait indefinitely until one decides to put the $\mathrm{CD}$ in a drive: time then becomes real and follows its arrow.

We think that imaginary time and, by extension, complex space-time coordinates, are one of the most promising tracks for generalizing relativistic quantum mechanics.

One of the advances of complex numbers in this direction is the concept of Minkowski complex space-time. A first attempt with emphasis on specific aspects of twistors has been performed in [6]. In the present paper, we come back to this idea but with a different metric. In fact, the metric considered in [6] involved a complex space-time interval while we want to limit ourselves to a real one. We could compare this to the wavefunction role in usual quantum mechanics. Indeed this function, although complex, appears on a real form, like the square of its modulus, when physics concepts must be discussed.

More precisely, the key idea involved by our proposal of $(3+1)$-D complex Minkowski manifold is to extend spatial as well as time coordinates to complex numbers with a Hermitian metric given by

$$
\mathrm{d} s^{2}=\eta_{\mu v} \overline{\mathrm{d} x^{\mu}} \mathrm{d} x^{v}
$$

with

$$
\eta=\operatorname{diag}(1,-1,-1,-1) ; x^{\mu}=\{t, x, y, z\} ; \mu, v=0,1,2,3
$$

Here the notation $\overline{x^{\mu}}$ refers to the complex conjugate of $x^{\mu}$ while the diag notation means that we consider a diagonal matrix.

The purpose of this paper is to investigate this $(3+1)$-D Minkowski manifold and, in particular, to see what would the Poincaré group/algebra become when coordinates are complex numbers.

To do so, we first have to consider a $(6+2)$-D real manifold by considering real as well as imaginary parts of the involved complex numbers. This is the subject of the next Section. Then, we use the corresponding coadjoint action to put in evidence the transformation laws on momenta which are nothing else than basis operators for the algebra. Sections 4 and 5 are devoted to the differential realization of these momenta in the real as well as the complex cases. The trans- 
formations of the group are then available in Section 6. We point out the Casimir operators in Section 7 and finally conclude in Section 8.

\section{The Extended Poincaré Group/Algebra}

As well known the Lorentz group $O(1,3)$ preserves

$$
X^{\mathrm{T}} \eta X
$$

where $\eta$ has been defined in (3) while

$$
X^{\mathrm{T}}=(t, x, y, z)=\left\{x^{\mu}, \mu=0,1,2,3\right\}
$$

( $X^{\mathrm{T}}$ refers here to the transposition of $X$ ).

If we now consider complex coordinates $x^{\mu}$, Equation (4) is replaced by $\left(X_{C}^{\dagger} \equiv\left(\overline{X_{C}}\right)^{\mathrm{T}}\right)$

$$
X_{C}^{\dagger} \eta X_{C} ; X_{C}^{\mathrm{T}}=(t, x, y, z)=\left\{x^{\mu}, \mu=0,1,2,3\right\}
$$

The Lie group $U(1,3, C)$ is, by definition, the one leaving the quadratic form (5) invariant.

This quadratic form (5) is equivalent to the orthogonal one

$$
X_{R}^{\mathrm{T}} G X_{R}
$$

with

$$
X_{R}^{\mathrm{T}}=\left\{x_{R}^{\mu}, x_{I}^{\mu}, \mu=0,1,2,3\right\}=\left\{x^{A}, A=0,1,2, \cdots, 7\right\}
$$

and

$$
G=\operatorname{diag}(1,-1,-1,-1,1,-1,-1,-1)
$$

We obviously have

$$
x^{\mu}=x_{R}^{\mu}+i x_{I}^{\mu}
$$

The Lie group preserving (6) is $O(2,6)$.

Even if the quadratic forms are formally equivalent, the two groups $U(1,3, C)$ and $O(2,6)$ have a different number of parameters $(16$ for $U(1,3, C)$ and 29 for $O(2,6))$. This finding, that might be surprising at first sight, will be explained in a next Section.

Due to the embedding of $U(1,3, C)$ in $O(2,6)$, we focus on the larger group.

Consequently, we define the extended Poincare group on the $(6+2)$-D real Minkowski space as the set of the following ( 9 by 9) matrices

$$
g=\left(\begin{array}{cc}
L & \alpha \\
0 & 1
\end{array}\right)
$$

where the 8-vector

$$
\alpha^{\mathrm{T}}=\left(\alpha^{A}, A=0,1,2, \cdots, 7\right)
$$

is associated with (real) translations in the ( $6+2)$-D manifold and $L$ is the ( 8 by 8) matrix of the $O(2,6)$ group i.e. 


$$
G L^{\mathrm{T}} G L=I
$$

The Lie algebra corresponding to (10) is the vector space of the matrices given by

$$
Z=\left(\begin{array}{cc}
\omega & \gamma \\
0 & 0
\end{array}\right) ; \gamma^{\mathrm{T}}=\left(\gamma^{A}, A=0,1,2, \cdots, 7\right)
$$

Here the coefficients $\gamma^{A}$ are real numbers and $\omega$ is the matrix of the $\operatorname{so}(2,6)$ algebra defined by

$$
G \omega^{\mathrm{T}} G=-\omega
$$

In details, we have

$$
\omega=\sum_{A, B=0}^{7} J_{A B} j^{A B}
$$

where $J_{A B}$ are real numbers and $j^{A B}$ are the (28) basis matrices of $\operatorname{so}(2,6)$ namely

$$
j^{A B}=\varepsilon(A B) e^{A B}-\varepsilon(B A) e^{B A}
$$

(no summation on repeated indices). In Equation (15), the numbers $\varepsilon(A B)(= \pm 1)$ are constrained by

$$
\varepsilon(A B) \varepsilon(C D) \delta^{B C}=G^{B C} \varepsilon(A D) ; \varepsilon(A B) \varepsilon(C D) \delta^{A D}=G^{A D} \varepsilon(C B)
$$

while the notation $e^{A B}$ stands for a 8 by 8 matrix made of " 0 " everywhere except a " 1 " at the intersection of the $(A+1)^{\text {th }}$ line and the $(B+1)^{\text {th }}$ column.

Noticing that

$$
e^{A B} e^{C D}=\delta^{B C} e^{A D}
$$

we come easily to the $s o(2,6)$ commutation relations

$$
\left[j^{A B}, j^{C D}\right]=G^{B C} j^{A D}+G^{B D} j^{C A}+G^{A C} j^{D B}+G^{A D} j^{B C} ; j^{A B}=-j^{B A}
$$

We will come back to this algebra in Section 4. Let us just conclude this one by mentioning that the adjoint representation of the extended Poincaré group is, by definition, given by

$$
Z^{\prime}=g Z g^{-1}
$$

This leads to

$$
\omega^{\prime}=L \omega L^{-1}
$$

as well as to the following relation

$$
\gamma^{\prime}=-L \omega L^{-1} \alpha+L \omega
$$

\section{The Coadjoint Representation: Transformation Laws of the Momenta}

Here we follow Souriau's approach [7], one of us has already successfully applied it [8] to Kaluza 5-D space-time. By analogy with what has been done in the so $(1,3)$ case, we define a torsor $\mu$ of the extended Poincare group by the 
identity

$$
\mu(Z) \equiv \frac{1}{2} \operatorname{Tr}(M \omega)+(G P)^{\mathrm{T}} \gamma
$$

where

$$
\mu \equiv\{P, M\}, P \in \mathbf{R}^{8}, G M^{\mathrm{T}} G=-M
$$

We require the invariance

$$
\mu^{\prime}\left(Z^{\prime}\right)=\mu(Z)
$$

or, in other words

$$
\frac{1}{2} \operatorname{Tr}(M \omega)+(G P)^{\mathrm{T}} \gamma=\frac{1}{2} \operatorname{Tr}\left(M^{\prime} L \omega L^{-1}\right)+P^{\prime \mathrm{T}} G\left(-L \omega L^{-1} \alpha+L \omega\right)
$$

where we have used Equations (20)-(21) of the adjoint representation.

The relation (25) implies the following transformation on the momenta P:

$$
P^{\prime}=L P
$$

Thus, Equation (25) reduces to

$$
\frac{1}{2} \operatorname{Tr}(M \omega)=\frac{1}{2} \operatorname{Tr}\left(M^{\prime} L \omega L^{-1}\right)-P^{\mathrm{T}} G \omega L^{-1} \alpha
$$

Remembering that the last term is the product of a transposed 8-vector and an 8 -vector, we can rewrite this relation as

$$
\frac{1}{2} \operatorname{Tr}\left(M^{\prime} L \omega L^{-1}\right)=\frac{1}{2} \operatorname{Tr}(M \omega)+\operatorname{Tr}\left(G \omega L^{-1} \alpha P^{\mathrm{T}}\right)
$$

which, after usual manipulations on the trace, leads to

$$
\frac{1}{2} \operatorname{Tr}\left(L^{-1} M^{\prime} L \omega\right)=\frac{1}{2} \operatorname{Tr}(M \omega)+\operatorname{Tr}\left(L^{-1} \alpha P^{\mathrm{T}} G \omega\right)
$$

or, in an equivalent way

$$
\operatorname{Tr}\left(L^{-1} M^{\prime} L \omega\right)=\operatorname{Tr}(M \omega)+\operatorname{Tr}\left(L^{-1} \alpha P^{\mathrm{T}} G \omega\right)-\operatorname{Tr}\left(P \alpha^{\mathrm{T}} G L \omega\right)
$$

We thus come to the conclusion that

$$
M^{\prime}=L M G L^{\mathrm{T}} G+\alpha P^{\mathrm{T}} L^{\mathrm{T}} G-L P \alpha^{\mathrm{T}} G
$$

Equations (26) and (28) provide the transformation laws of the momenta.

\section{Differential Realization of the Momenta: The (6+2)-D Real Poincaré Algebra}

Let us take a look at the result (26). If we write the transformations on the coordinates through the group as (cf. Equation (10))

$$
X^{\prime}=L X+\alpha
$$

they can be inversed following

$$
X=G L^{\mathrm{T}} G X^{\prime}-G L^{\mathrm{T}} G \alpha
$$

This implies that

$$
\nabla^{\prime}=G L G \nabla
$$


where $\nabla$ is the $8 \mathrm{D}$-gradient

$$
\nabla^{\mathrm{T}}=\left\{\frac{\partial}{\partial x^{A}}, A=0,1,2, \cdots, 7\right\}
$$

Comparing with (26), it is obvious to conclude that

$$
P=C G \nabla(C=\text { constant })
$$

With the conventions (7) and

$$
\partial_{A} \equiv \frac{\partial}{\partial x^{A}}
$$

the momenta $P$ are thus

$$
P_{A}=C \partial_{A} ; P^{A}=C \partial^{A}=C G^{A B} P_{B}
$$

We recover the momenta of usual Quantum Mechanics if the constant $C$ is fixed as $C=i \bar{h}$ and $A$ limited to the first four values. However, for simplicity, we fix here $C=1$ so that

$$
P_{A}=\partial_{A} ; P^{A}=G^{A B} P_{B}
$$

In the same way, the momenta $M$ satisfying (28) can be realized through a matrix similar to $\omega$ (see Equation (14))

$$
M=\sum_{A, B=0}^{7} J_{A B} j^{A B}
$$

but with

$$
J^{A B}=x^{A} \partial^{B}-x^{B} \partial^{A}, A, B=0,1,2, \cdots, 7
$$

In other words, we have

$$
M=X P^{\mathrm{T}} G-P X^{\mathrm{T}} G
$$

And it is straightforward to convince ourselves of (28) to be satisfied by using (26) and (29).

It is then easy to find out the commutation relations of the $(6+2)$-D Poincaré algebra by using

$$
\left[x^{A}, \partial^{B}\right]=-G^{A B}
$$

They read

$$
\left[J^{A B}, J^{C D}\right]=G^{B C} J^{A D}+G^{B D} J^{C A}+G^{A C} J^{D B}+G^{A D} J^{B C}
$$

in agreement with Equation (18) and

$$
\begin{gathered}
{\left[J^{A B}, P^{C}\right]=-G^{A C} P^{B}+G^{B C} P^{A}} \\
{\left[P^{A}, P^{B}\right]=0}
\end{gathered}
$$

\section{The $(3+1)$-D Complex Poincaré Algebra}

By making use of the change of variables (9), we can define linear combinations of the $J^{A B}$ as well as the $P^{A}$ operators in order to restore the extended Poincaré algebra in a $(3+1)$-D complex manifold. These linear combinations write 


$$
\begin{gathered}
M^{\mu v}=-J^{\mu v}-J^{(\mu+4)(v+4)} \\
M_{I}^{\mu \nu}=-J^{\mu(v+4)}-J_{v(\mu+4)} \\
N^{\mu v}=-J^{\mu v}+J^{(\mu+4)(v+4)} \\
N_{I}^{\mu \nu}=-J^{\mu(v+4)}+J_{v(\mu+4)} \\
P_{C}^{\mu}=\frac{1}{2}\left(P^{\mu}-i P^{\mu+4}\right) ; \overline{P_{C}^{\mu}}=\frac{1}{2}\left(P^{\mu}+i P^{\mu+4}\right)
\end{gathered}
$$

They lead to the following realizations in terms of complex coordinates $x^{\mu}$ and their derivatives

$$
\begin{gathered}
\partial_{\mu}=\frac{\partial}{\partial x^{\mu}}=\left(\frac{\partial}{\partial t}, \frac{\partial}{\partial x}, \frac{\partial}{\partial y}, \frac{\partial}{\partial z}\right), \partial^{\mu}=\frac{\partial}{\partial x_{\mu}}=\left(\frac{\partial}{\partial t},-\frac{\partial}{\partial x},-\frac{\partial}{\partial y},-\frac{\partial}{\partial z}\right) \\
M^{\mu v}=-x^{\mu} \partial^{v}+x^{v} \partial^{\mu}-\overline{x^{\mu} \partial^{v}}+\overline{x^{v} \partial^{\mu}} \\
M_{I}^{\mu \nu}=-i x^{\mu} \partial^{v}-i x^{v} \partial^{\mu}+\overline{i x^{\mu} \partial^{v}}+i \overline{x^{v} \partial^{\mu}} \\
N^{\mu v}=-\overline{x^{\mu}} \partial^{v}+\overline{x^{v}} \partial^{\mu}-x^{\mu} \overline{\partial^{v}}+x^{v} \overline{\partial^{\mu}} \\
N_{I}^{\mu v}=-i \overline{x^{\mu}} \partial^{v}+\overline{i x^{v}} \partial^{\mu}+i x^{\mu} \overline{\partial^{v}}-i x^{v} \overline{\partial^{\mu}} \\
P_{C}^{\mu}=\partial^{\mu}, \overline{P_{C}^{\mu}}=\overline{\partial^{\mu}}
\end{gathered}
$$

It was already evident through Equations (40) but it is even more obvious here that $M^{\mu \nu}, N^{\mu \nu}, N_{I}^{\mu \nu}$ are antisymmetric on their indices and thus there are six of them for each category while $M_{I}^{\mu v}$ is symmetric leading to ten different operators. These 28 operators are real ones: only the four $P_{C}^{\mu}$ are complex and have to be supplemented by their conjugates.

The corresponding commutation relations are then

$$
\begin{aligned}
& {\left[M^{\mu v}, M^{\alpha \beta}\right]=\eta^{\alpha v} M^{\beta \mu}+\eta^{\beta v} M^{\mu \alpha}+\eta^{\alpha \mu} M^{v \beta}+\eta^{\beta \mu} M^{\alpha v}} \\
& {\left[M^{\mu v}, M_{I}^{\alpha \beta}\right]=-\eta^{\alpha v} M_{I}^{\beta \mu}-\eta^{\beta v} M_{I}^{\mu \alpha}+\eta^{\alpha \mu} M_{I}^{v \beta}+\eta^{\beta \mu} M_{I}^{\alpha v}} \\
& {\left[M_{I}^{\mu v}, M_{I}^{\alpha \beta}\right]=-\eta^{\alpha v} M^{\beta \mu}+\eta^{\beta v} M^{\mu \alpha}+\eta^{\alpha \mu} M^{v \beta}-\eta^{\beta \mu} M^{\alpha v}} \\
& {\left[M^{\mu v}, N^{\alpha \beta}\right]=\eta^{\alpha v} N^{\beta \mu}+\eta^{\beta v} N^{\mu \alpha}+\eta^{\alpha \mu} N^{v \beta}+\eta^{\beta \mu} N^{\alpha v}} \\
& {\left[M_{I}^{\mu v}, N^{\alpha \beta}\right]=-\eta^{\alpha v} N_{I}^{\beta \mu}-\eta^{\beta v} N_{I}^{\mu \alpha}+\eta^{\alpha \mu} N_{I}^{v \beta}+\eta^{\beta \mu} N_{I}^{\alpha v}} \\
& {\left[N^{\mu v}, N^{\alpha \beta}\right]=\eta^{\alpha v} M^{\beta \mu}+\eta^{\beta v} M^{\mu \alpha}+\eta^{\alpha \mu} M^{v \beta}+\eta^{\beta \mu} M^{\alpha v}} \\
& {\left[M^{\mu v}, N_{I}^{\alpha \beta}\right]=\eta^{\alpha v} N_{I}^{\beta \mu}+\eta^{\beta v} N_{I}^{\mu \alpha}+\eta^{\alpha \mu} N_{I}^{v \beta}+\eta^{\beta \mu} N_{I}^{\alpha v}} \\
& {\left[M_{I}^{\mu v}, N_{I}^{\alpha \beta}\right]=\eta^{\alpha v} N^{\beta \mu}+\eta^{\beta v} N^{\mu \alpha}-\eta^{\alpha \mu} N^{v \beta}-\eta^{\beta \mu} N^{\alpha v}} \\
& {\left[N^{\mu v}, N_{I}^{\alpha \beta}\right]=-\eta^{\alpha v} M_{I}^{\beta \mu}+\eta^{\beta v} M_{I}^{\mu \alpha}+\eta^{\alpha \mu} M_{I}^{v \beta}-\eta^{\beta \mu} M_{I}^{\alpha v}} \\
& {\left[N_{I}^{\mu v}, N_{I}^{\alpha \beta}\right]=\eta^{\alpha v} M^{\beta \mu}+\eta^{\beta v} M^{\mu \alpha}+\eta^{\alpha \mu} M^{v \beta}+\eta^{\beta \mu} M^{\alpha v}} \\
& {\left[M^{\mu v}, P_{C}^{\alpha}\right]=\eta^{\alpha \mu} P_{C}^{v}-\eta^{\alpha v} P_{C}^{\mu} ;\left[M^{\mu v}, \overline{P_{C}^{\alpha}}\right]=\eta^{\alpha \mu} \overline{P_{C}^{v}}-\eta^{\alpha v} \overline{P_{C}^{\mu}}}
\end{aligned}
$$




$$
\begin{aligned}
& {\left[M_{I}^{\mu \nu}, P_{C}^{\alpha}\right]=i \eta^{\alpha \mu} P_{C}^{v}+i \eta^{\alpha v} P_{C}^{\mu} ;\left[M_{I}^{\mu \nu}, \overline{P_{C}^{\alpha}}\right]=-i \eta^{\alpha \mu} \overline{P_{C}^{v}}-i \eta^{\alpha \nu} \overline{P_{C}^{\mu}}} \\
& {\left[N^{\mu \nu}, P_{C}^{\alpha}\right]=\eta^{\alpha \mu} \overline{P_{C}^{v}}-\eta^{\alpha \nu} \overline{P_{C}^{\mu}} ;\left[N^{\mu \nu}, \overline{P_{C}^{\alpha}}\right]=\eta^{\alpha \mu} P_{C}^{v}-\eta^{\alpha \nu} P_{C}^{\mu}} \\
& {\left[N_{I}^{\mu \nu}, P_{C}^{\alpha}\right]=-i \eta^{\alpha \mu} \overline{P_{C}^{v}}+i \eta^{\alpha \nu} \overline{P_{C}^{\mu}} ;\left[N_{I}^{\mu \nu}, \overline{P_{C}^{\alpha}}\right]=i \eta^{\alpha \mu} P_{C}^{v}-i \eta^{\alpha v} P_{C}^{\mu}} \\
& {\left[P_{C}^{\alpha}, P_{C}^{\beta}\right]=\left[P_{C}^{\alpha}, \overline{P_{C}^{\beta}}\right]=\left[\overline{P_{C}^{\alpha}} \overline{P_{C}^{\beta}}\right]=0}
\end{aligned}
$$

The usual Poincare algebra is recovered through Equations (42a), (43k) and (43o). It is now realized through complex variables and corresponds to the proposal made by one of us in [8]. We can also recognize the algebra $u(1,3, C)$ through the operators (42a)-(42b) and their commutation relations (43a)-(43c). The operators (42c)-(42d) are the complement of $u(1,3, C)$ in $s o(2,6)$.

\section{Transformations of the Extended Poincaré Group}

Now that the algebraic content is clear, let us come to the group approach subtended by the transformations (29).

We recognize in (29) eight real translations associated with $\alpha$, or, in an equivalent way, four complex ones. The contributions of the matrix $L$ can be understood as follows:

1) Twelve transformations of "boost" type i.e. "rotations" between a (real or imaginary) time component and three (real or imaginary) space components:

$$
\left\{\begin{array}{l}
t_{a}^{\prime}=\cosh \left(\theta_{a b}^{0 j}\right) t_{a}-\sinh \left(\theta_{a b}^{0 j}\right) x_{b}^{j} \\
x_{b}^{\prime j}=-\sinh \left(\theta_{a b}^{0 j}\right) t_{a}+\cosh \left(\theta_{a b}^{0 j}\right) x_{b}^{j}
\end{array}\right.
$$

with $j=1,2,3 ; a=R, I ; b=R, I$.

2) Twelve rotations between (real or imaginary) space components:

$$
\left\{\begin{array}{l}
x_{a}^{\prime k}=\cos \left(\theta_{a b}^{j k}\right) x_{a}^{k}+\sin \left(\theta_{a b}^{j k}\right) x_{b}^{j} \\
x_{b}^{\prime j}=-\sin \left(\theta_{a b}^{j k}\right) x_{a}^{k}+\cos \left(\theta_{a b}^{j k}\right) x_{b}^{j}
\end{array}\right.
$$

with $j=1,2,3, j \neq k ; a=R, I ; b=R, I$.

3) Four rotations between real and imaginary parts of one of the components:

$$
\begin{gathered}
\left\{\begin{array}{l}
t_{R}^{\prime}=\cos \left(\theta^{00}\right) t_{R}-\sin \left(\theta^{00}\right) t_{I} \\
t_{I}^{\prime}=\sin \left(\theta^{00}\right) t_{R}+\cos \left(\theta^{00}\right) t_{I}
\end{array}\right. \\
\left\{\begin{array}{l}
x_{R}^{\prime j}=\cos \left(\theta^{i j}\right) x_{R}^{j}+\sin \left(\theta^{i j}\right) x_{I}^{j} \\
x_{I}^{\prime j}=-\sin \left(\theta^{i j}\right) x_{R}^{j}+\cos \left(\theta^{i j}\right) x_{I}^{j}
\end{array}\right.
\end{gathered}
$$

Let us rewrite these results within the complex coordinates:

$$
t^{\prime}=e^{i \theta^{00}} t ; x^{\prime j}=e^{-i \theta^{i j}} x^{j}
$$

These equations are remarkable in the sense that they show that the complexification enables the connection between the Lorentz components. Indeed we know [9] that the Lorentz group has four components: $L_{+}^{\uparrow}, L_{-}^{\uparrow}=P L_{+}^{\uparrow}, L_{+}^{\downarrow}=P T L_{+}^{\uparrow}$ 
and $L_{-}^{\downarrow}=T L_{+}^{\uparrow}$. Each of the three last ones is related to the first one by acting on it with a discrete symmetry, either the parity operator $P$ or the time-reversal operator $T$. Two objects in "mirror symmetry" are necessarily linked by a rotation in a higher dimensional space and that's exactly what happens here: the complexification allows these dimensions to exist in order to relate a coordinate and its opposite (which corresponds to $\theta^{\mu \mu}=\pi$ ).

Similar discussions can be made with the complex algebra. We will just mention here that if the $U(1,3, C)$ operators perform transformations such as boosts or rotations on the four complex coordinates $x^{\mu}$, the other 12 ones (corresponding to exponentiations of $N^{\mu v}$ or $N_{I}^{\mu v}$ ) realize, in addition, a complex conjugation. It is actually the reason why, despite of the fact that the quadratic forms (5) and (6) are equivalent, the Lie groups $U(1,3, C)$ and $O(2,6)$ are not isomorphic.

\section{The Casimir Operators}

The number of Casimir operators associated with the inhomogeneous group $\operatorname{ISO}(2,6)$ (which is the one subtended by our approach in the real space) is equal to 4 [10].

We put here explicitely in evidence two of them i.e. the ones generalizing the two Casimirs of the Poincaré algebra.

The first Casimir operator is

$$
C_{1}=P^{\mathrm{T}} G P
$$

It is indeed invariant under the transformation (26). Another way to be convinced of the form (48) is to rewrite it in details

$$
C_{1}=P^{A} P_{A}=4 P_{C}^{\mu} P_{C \mu}
$$

and verify that it commutes with each of the 36 operators of the extended Poincaré algebra by using Equations (38)-(39) or (43k)-(43o) depending on whether we choose to work with eight real coordinates or four complex ones.

By analogy with the real case, we can thus define the mass of a particle living in the complex Minkowski space-time by

$$
\left|E^{2}\right|-\left|\boldsymbol{p}^{2}\right|=m^{2} ; E=P_{C}^{0}, p^{j}=P_{C}^{j}
$$

a relation which simplifies when the system is at rest and gives

$$
E=m e^{i \phi}
$$

This is the complex version of the famous Einstein relation ${ }^{2} E=m$.

The second Casimir operator requests more calculations. We indeed need to introduce the following 3-rank tensor

$$
W^{A B C}=J^{A B} P^{C}-J^{A C} P^{B}+J^{B C} P^{A}
$$

It is antisymmetric on the two first indices as well as on the two last ones but is (evidently) symmetric on $A$ and $C$. It thus gives rise to 56 operators.

${ }^{2}$ Recall that $c=1$. 
Let us take a while to mention that, in the real case, these operators reduce to the four well known ones

$$
W_{\mu}=\frac{1}{2} \varepsilon_{\mu \nu \lambda \alpha} J^{\nu \lambda} P^{\alpha}
$$

namely the four components of the Pauli-Lubanski pseudo-vector. No need to say that, here, there is no interest to go through the dual to obtain a similar writing, as this will lead to a pseudo-tensor of rank 5.

So let us focus on the operators (52). It is easy (even if laborious) to convince ourselves that they are such that

$$
\begin{aligned}
{\left[W^{A B C}, J^{D E}\right]=} & G^{A D} W^{B C E}-G^{A E} W^{B C D}-G^{B D} W^{A C E} \\
& +G^{B E} W^{A C D}+G^{C D} W^{A B E}-G^{C E} W^{A B D} \\
& {\left[W^{A B C}, P^{D}\right]=0 }
\end{aligned}
$$

and

$$
\begin{aligned}
& {\left[W^{A B C}, W^{D E F}\right]} \\
& =G^{A D}\left(W^{B C E} P^{F}-W^{B C F} P^{E}\right)+G^{A E}\left(W^{B C F} P^{D}-W^{B C D} P^{F}\right) \\
& +G^{A F}\left(W^{B C D} P^{E}-W^{B C E} P^{D}\right)+G^{B D}\left(W^{A E F} P^{C}-W^{C E F} P^{A}\right) \\
& \quad+G^{B E}\left(-W^{A D F} P^{C}+W^{C D F} P^{A}\right)+G^{B F}\left(W^{A C E} P^{D}-W^{A C D} P^{E}\right) \\
& +G^{C D}\left(-W^{A B F} P^{E}+W^{A B E} P^{F}\right)+G^{C E}\left(-W^{A B D} P^{F}+W^{A B F} P^{D}\right) \\
& +G^{C F}\left(W^{D A B} P^{E}-W^{E A B} P^{D}\right)
\end{aligned}
$$

The two first relations show that

$$
C_{2}=W^{A B C} W_{A B C}
$$

does commute with each of the 36 generators of the extended Poincare algebra and, consequently, is the second Casimir operator.

Its interpretation goes through the so-called "little groups" technics i.e. restricting ourselves to particular momenta $P$. If the rest is considered, only two $P$ operators do not vanish:

$$
P^{0}=2 m \cos \phi ; P^{4}=2 m \sin \phi
$$

This implies the following form for $C_{2}$

$$
\begin{aligned}
C_{2}= & 4 m^{2}\left(J_{12}^{2}+J_{31}^{2}+J_{23}^{2}+J_{15}^{2}+J_{16}^{2}+J_{17}^{2}+J_{25}^{2}\right. \\
& \left.+J_{26}^{2}+J_{27}^{2}+J_{35}^{2}+J_{36}^{2}+J_{37}^{2}+J_{56}^{2}+J_{57}^{2}+J_{67}^{2}\right) \\
& -4 m^{2}(\cos \phi)^{2}\left(J_{14}^{2}+J_{24}^{2}+J_{34}^{2}+J_{45}^{2}+J_{46}^{2}+J_{47}^{2}\right) \\
& -4 m^{2}(\sin \phi)^{2}\left(J_{01}^{2}+J_{02}^{2}+J_{03}^{2}+J_{05}^{2}+J_{06}^{2}+J_{07}^{2}\right) \\
& -4 m^{2} \sin \phi \cos \phi\left(\left\{J_{01}, J_{14}\right\}+\left\{J_{02}, J_{24}\right\}+\left\{J_{03}, J_{34}\right\}\right. \\
& \left.+\left\{J_{05}, J_{45}\right\}+\left\{J_{06}, J_{46}\right\}+\left\{J_{07}, J_{47}\right\}\right)
\end{aligned}
$$

We notice that the only $f^{A B}$ that does not appear in (57) is $f^{44}$ namely the rotation on time. We also recover the spin interpretation of the real case except that we 
have here 20 possibilities for one spin algebra $(s o(3))$ to be put in evidence. This is evidently due to the increasing of spatial dimensions which are now 6 .

\section{Conclusions}

We have extended the Poincaré group as well as its algebra to a complex Minkowski space. Beyond the real operators corresponding to transformations of the real coordinates, our approach shows that supplementary imaginary as well as complex operators appear to form with the previous ones a 36-dimensional real or complex algebra according to choosing 8 real coordinates or 4 complex ones.

What are the unirreps of the extended Poincare algebra? What physical implications could have this complex algebra? What would be a complex version of usual quantum mechanics?

These questions remain open at this stage and will be the subject of further considerations.

\section{Data Availability}

The data that support the findings of this study are available within this article.

\section{Conflicts of Interest}

The authors declare no conflicts of interest regarding the publication of this paper.

\section{References}

[1] Petiau, G. (1936) Classe des Sciences, 8, 16.

[2] Velo, G. and Zwanziger, D. (1969) Physical Review, 186, 1337.

[3] Hartle, J.B. and Hawking, S.W. (1983) Physical Review D, 28, 2960. https://doi.org/10.1103/PhysRevD.28.2960

[4] Deltete, R.J. and Guy, R.A. (1996) Synthese, 108, 185-203. https://doi.org/10.1007/BF00413497

[5] Bogdanoff, G. and Bogdanoff, I. (2002) Annals of Physics, 296, 90-97. https://doi.org/10.1006/aphy.2001.6212

[6] Hansen, R.O., Newman, E.T., Penrose, R. and Tod, K.P. (1978) Proceedings of the Royal Society of London A, 363, 445-468. https://doi.org/10.1098/rspa.1978.0177

[7] Souriau, J.M. (1997) Structure of Dynamical Systems. In: Progress in Mathematics, Springer, Boston, Vol. 149. https://doi.org/10.1007/978-1-4612-0281-3

[8] Petit, J.P. (2018) Progress in Physics, 14, 38.

[9] Srinivasa Rao, K.N. (1988) The Rotation and Lorentz Groups and Their Representations for Physicists. Wiley, New-Delhi, p. 213.

[10] Chaichian, M., Demichev, A.P. and Nelipa, N.F. (1983) Communications in Mathematical Physics, 90, 353-372. https://doi.org/10.1007/BF01206887 